\title{
Validation of multiparametric MRI by histopathology after nephrectomy: a case study
}

\author{
Anneloes de Boer ${ }^{1}$ (D) Tobias T. Pieters ${ }^{2} \cdot$ Anita A. Harteveld ${ }^{1} \cdot$ Peter J. Blankestijn ${ }^{2} \cdot$ Clemens Bos $^{1}$. \\ Martijn Froeling ${ }^{1} \cdot$ Roel Goldschmeding ${ }^{3} \cdot$ Hans J. M. Hoogduin ${ }^{1}$. Jaap A. Joles ${ }^{2}$ - Bart-Jeroen Petri ${ }^{4}$. \\ Marianne C. Verhaar ${ }^{2} \cdot \operatorname{Tim}_{\text {Leiner }}{ }^{1} \cdot$ Tri Q. Nguyen $^{3} \cdot$ Arjan D. van Zuilen $^{2}$
}

Received: 27 March 2020 / Revised: 23 July 2020 / Accepted: 1 September 2020 / Published online: 20 September 2020

(c) The Author(s) 2020

\begin{abstract}
Objectives Renal multiparametric MRI (mpMRI) is a promising tool to monitor renal allograft health to enable timely treatment of chronic allograft nephropathy. This study aims to validate mpMRI by whole-kidney histology following transplantectomy.

Materials and methods A patient with kidney transplant failure underwent mpMRI prior to transplantectomy. The mpMRI included blood oxygenation level-dependent (BOLD) MRI, $T_{1}$ and $T_{2}$ mapping, diffusion-weighted imaging (DWI), 2D phase contrast (2DPC) and arterial spin labeling (ASL). Parenchymal mpMRI measures were compared to normative values obtained in 19 healthy controls. Differences were expressed in standard deviations (SD) of normative values. The mpMRI measures were compared qualitatively to histology.

Results The mpMRI showed a heterogeneous parenchyma consistent with extensive interstitial hemorrhage on histology. A global increase in $T_{1}(+3.0 \mathrm{SD})$ and restricted diffusivity $(-3.6 \mathrm{SD})$ were consistent with inflammation and fibrosis. Decreased $\mathrm{T}_{2}(-1.8 \mathrm{SD})$ indicated fibrosis or hemorrhage. ASL showed diminished cortical perfusion $(-2.9 \mathrm{SD})$ with patent proximal arteries. 2DPC revealed a $69 \%$ decrease in renal perfusion. Histological evaluation showed a dense inflammatory infiltrate and fibrotic changes, consistent with mpMRI results. Most interlobular arteries were obliterated while proximal arteries were patent, consistent with ASL findings.
\end{abstract}

Discussion mpMRI findings correlated well with histology both globally as well as locally.

Keywords Multiparametric magnetic resonance imaging $\cdot$ Histology $\cdot$ Kidney $\cdot$ Kidney transplantation

Anneloes de Boer and Tobias Pieters contributed equally.

Electronic supplementary material The online version of this article (https://doi.org/10.1007/s10334-020-00887-9) contains supplementary material, which is available to authorized users.

Anneloes de Boer

a.deboer-13@umcutrecht.nl

1 Department of Radiology, University Medical Center Utrecht, Utrecht University, Utrecht, The Netherlands

2 Department of Nephrology and Hypertension, University Medical Center Utrecht, Utrecht University, Utrecht, The Netherlands

3 Department of Pathology, University Medical Center Utrecht, Utrecht University, Utrecht, The Netherlands

4 Department of Vascular Surgery, University Medical Center Utrecht, Utrecht University, Utrecht, The Netherlands

\section{Introduction}

Kidney transplantation is the treatment of choice for patients with end-stage kidney disease [1]. Close monitoring of the graft is crucial for early detection of treatable conditions [2]. Traditional methods of graft monitoring like the measurement of plasma creatinine and proteinuria only deviate when the majority of nephrons are irreversibly lost. Some centers use protocol biopsies to detect subclinical disease. However, transplant biopsies are invasive, carry the risk of sampling errors and are associated with a small risk of graft loss [3]. Therefore, protocol biopsies are not standard of care in all centers and are not suitable for monitoring disease progression. Multiparametric kidney MRI may help overcome these problems. MRI is sensitive to various relevant functional and structural markers like perfusion, oxygenation and fibrosis [4]. It can map the kidneys as a whole and is non-invasive, 
making it a suitable candidate for monitoring of local and global disease progression. For example, $T_{1}$ and the apparent diffusion coefficient (ADC) correlate with fibrosis on histology and could be used to predict current graft dysfunction and future eGFR decline [5-7]. Perfusion measurements can be performed without contrast agent using arterial spin labeling (ASL) and have been shown to correlate with reduced capillary density [7-9].

Several histological findings in protocol biopsies, such as interstitial fibrosis and tubular atrophy (IF/TA) and capillary rarefaction, strongly correlate to graft survival $[10,11]$. Both capillary rarefaction and IF/TA may be caused by a treatable condition, such as subclinical rejection or return of the original disease.

In our center, a multiparametric renal MRI protocol has recently been developed and evaluated [9]. The protocol consists of relaxometry ( $T_{1}$ and $T_{2}$ mapping), BOLD MRI, DWI, ASL and 2D phase contrast (2DPC) to measure renal blood flow (RBF). Normative values are available in 19 healthy volunteers [9].

Previous studies on correlation of MRI measures with histology used kidney biopsies for histological validation, but given the often heterogeneous distribution of damage, biopsies might not be representative for the entire kidney. We recently had the unique opportunity to perform multiparametric MRI in a patient scheduled for allograft explantation, which allowed for a comparison of MRI findings to whole-kidney histology and to evaluate MRI performance in identifying intra-organ heterogeneity. The objective of this study was to validate the comprehensive MRI protocol to whole-kidney histology using (1) a comparison of MRI findings to normative values in healthy controls and (2) a comparison between whole-kidney histology and local as well as global changes in MRI measures.

\section{Case description}

The patient was a 46-year-old female with an extensive history of liver fibrosis and end-stage kidney disease of unknown origin. Eleven years ago, she underwent a kidney transplantation with a kidney donated after circulatory death. Several creatinine rises in the following years were attributed to tacrolimus toxicity and acute rejection. Seven years post-transplantation, the patient started hemodialysis because of chronic rejection, likely caused by non-adherence to the immunosuppressive regimen. Eleven years posttransplantation, chronic hematuria and onset of new HLAantibodies suggested ongoing rejection for which she was treated with prednisolone. A urologic analysis did not suggest other causes for hematuria. Because of persistent pain in the region of the transplant kidney and persistent hematuria, it was decided to explant the allograft. During surgery, the kidney had to be cut from the surrounding capsule which led to minor superficial lesions and hemorrhaging.

Prior to surgery, informed consent for inclusion in an ongoing study on multparametric MRI in transplant kidneys was obtained and the MRI was scheduled 10 days before transplantectomy.

\section{Materials and methods}

\section{MRI acquisition and processing}

The MRI examination was performed on a 3T MR system (Ingenia, Philips Healthcare, Eindhoven, the Netherlands; software release 5.3.1). The patient was asked to avoid saltand protein-rich meals and to drink $2 \mathrm{~L}$ per $24 \mathrm{~h}$ of nonalcoholic liquids on the day of the scan to roughly standardize hydration and dietary conditions. The scan protocol, MR system and scan conditions were the same as described earlier [9]; therefore, the baseline results of that study could be used as normative values.

The scan protocol is described in detail elsewhere [9]. In short, it consisted of localizer images followed by an anatomical $T_{1}$-weighted Dixon. BOLD/ $R_{2}^{*}$ mapping was performed with a 15 -echo gradient echo sequence. Diffusion-weighted imaging was performed with a set of $b$ values and directions per $b$ value that allowed for both a DTI and an IVIM analysis. $T_{1}$ mapping was performed with a slice-cycled inversion recovery sequence and $T_{2}$ mapping was achieved using $T_{2}$ preparations. Single slice 2D phase contrast allowed for quantification of blood velocity in the (transplant) renal artery. ASL was performed using a flowattenuated inversion recovery scheme with four different inversion times. Total scan time was approximately $1 \mathrm{~h}$. Scan parameters are presented in Table 1.

Processing involved post hoc motion correction, fitting of the appropriate model and delineation of regions of interest (ROIs), avoiding areas affected by artifacts. Quality of all scans was assessed visually by an expert reader (AB, 5 years of experience in renal imaging) and scans of insufficient quality as assessed by visual inspection were excluded from analysis. In the patient, no discrimination between cortex and medulla was possible. Therefore, whole-parenchyma ROIs were used to allow for comparison between the patient and controls. ROI generation was semi-automated, using a combination of thresholding and k-means clustering [9]. For ROI generation in the graft, manual intervention was required since the normal anatomy was virtually lost. The collecting system could be identified on the anatomical scans and was excluded. The remaining parenchyma was entirely included. For detailed information on post-processing, see [9]. 


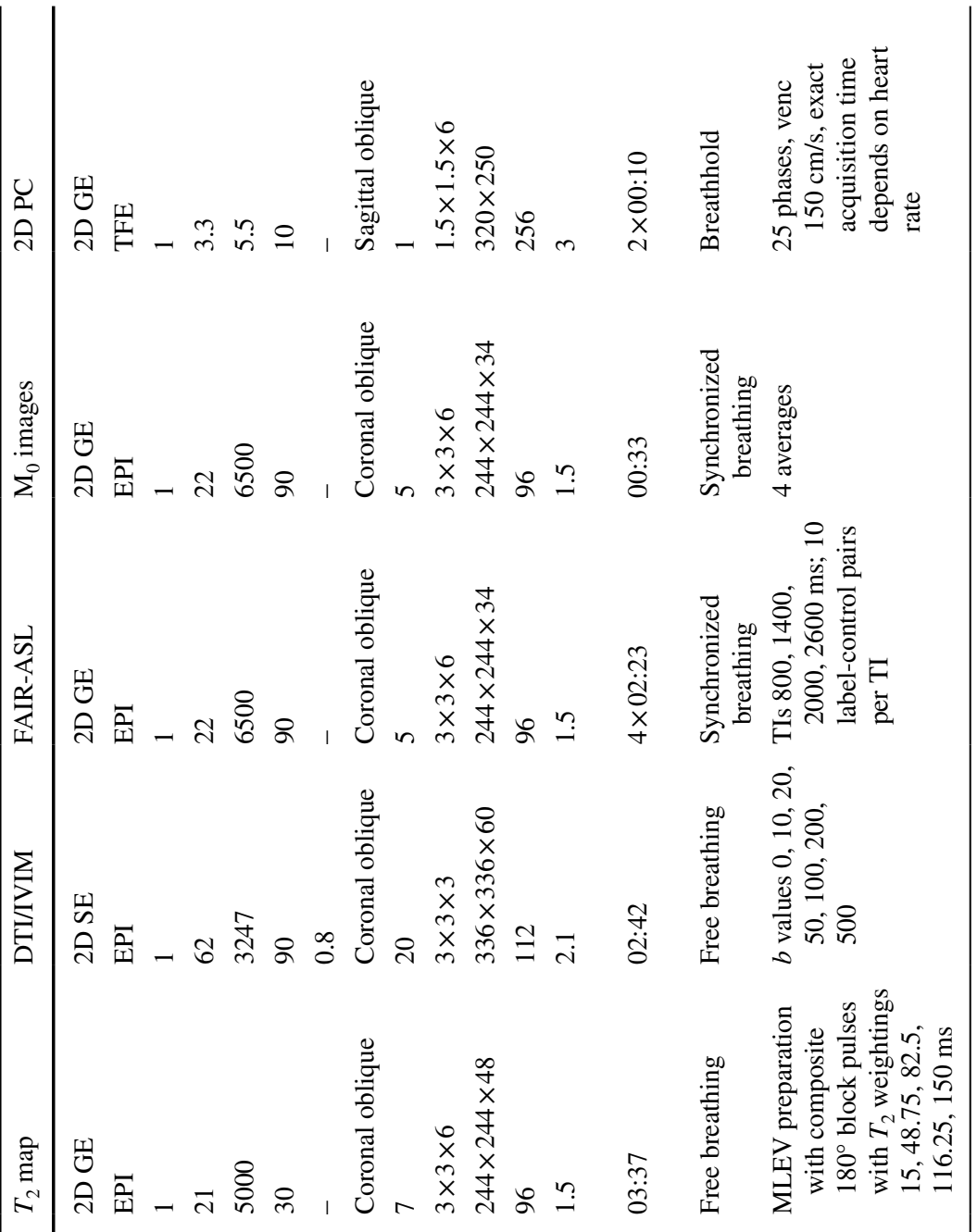

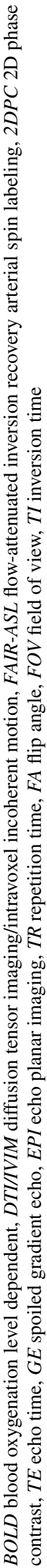



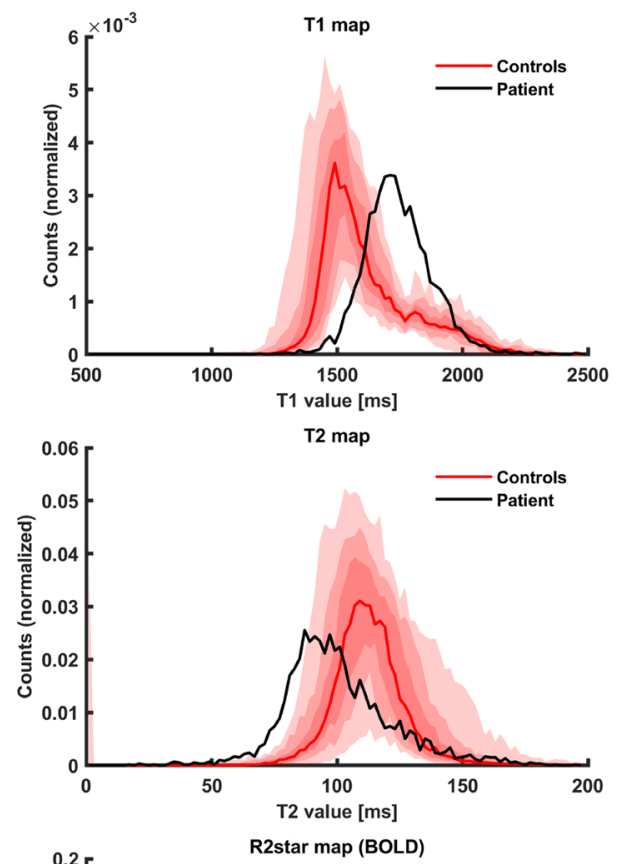

Patient Control
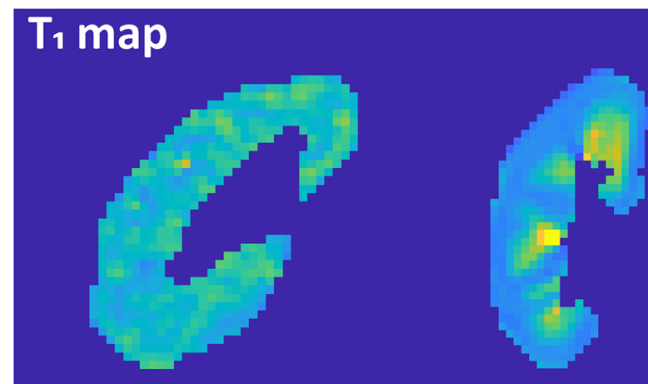

\section{$2500 \mathrm{~ms}$}
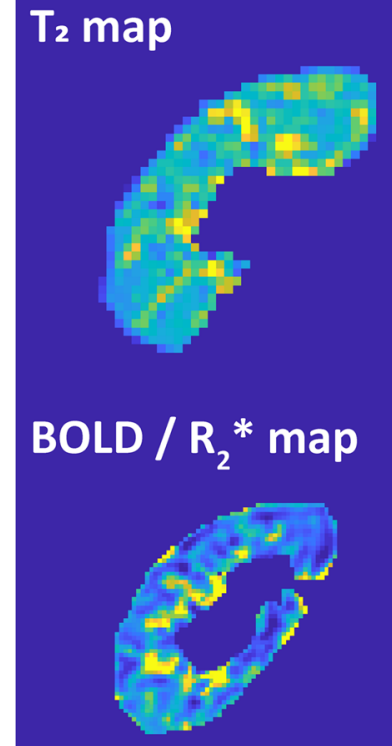

$50 \mathrm{~ms}$
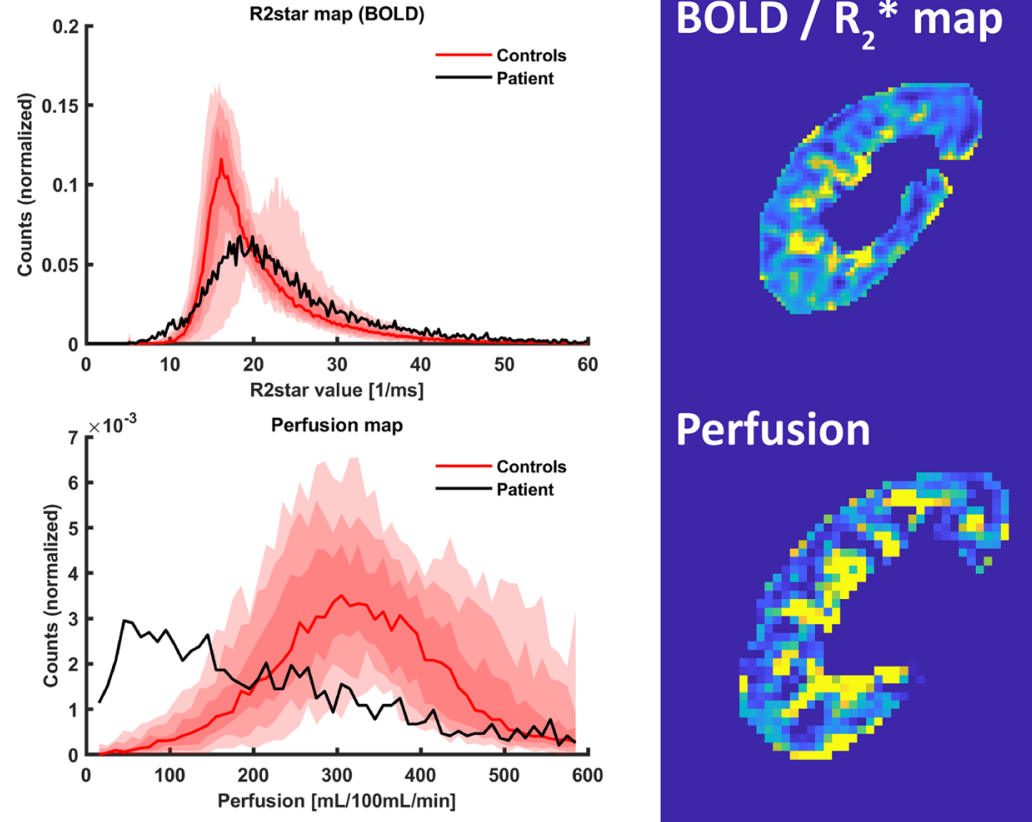

$10 \mathrm{~s}^{-1}$

$1000 \mathrm{~ms}$

$150 \mathrm{~ms}$

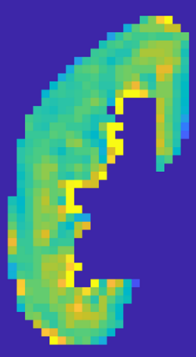

$50 \mathrm{~s}^{-1}$
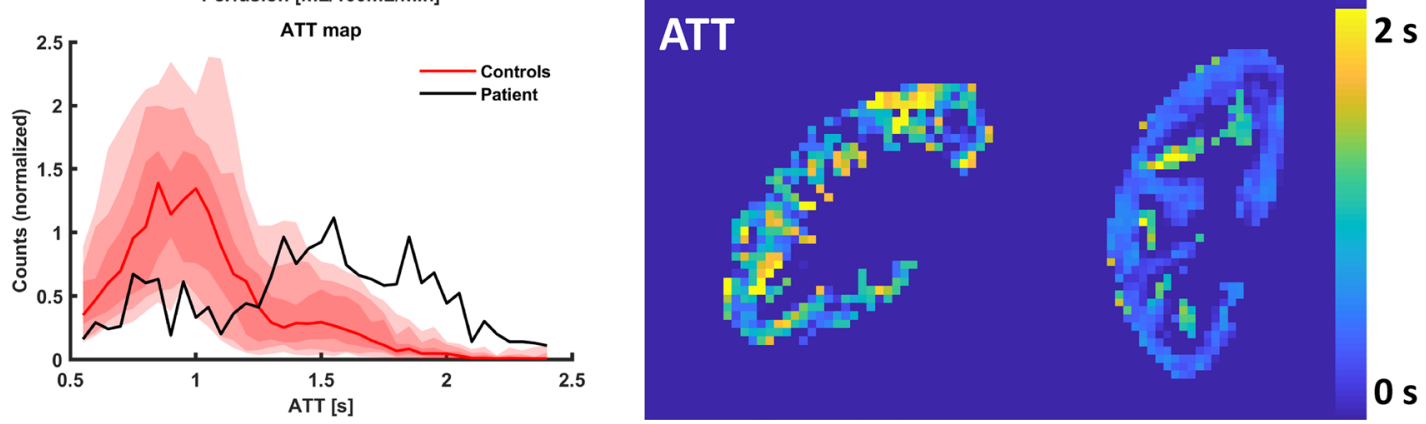
4Fig. 1 Histograms (left) and parameter maps for the patient (middle column) and a single control (right) for $T_{1}$ and $T_{2}$ mapping, BOLD and perfusion and ATT as assessed by ASL. The histograms show the voxel distribution in the patient and the median voxel distribution in controls, along with the spread between controls. The shaded areas denote the interquartile range, the 10-90 and the 2.5-97.5 interpercentile range (dark to lightly shaded). The histograms are normalized. $B O L D$ blood oxygenation level-dependent MRI, ATT arterial transit time, $A S L$ arterial spin labeling

After processing, several parameter maps were obtained. For relaxometry, $T_{1}, T_{2}$ and $R_{2}^{*}$ maps were generated. For the DWI data, both diffusion tensor imaging (DTI) and intravoxel incoherent motion (IVIM) analysis were performed. DTI analysis yielded the fractional anisotropy (FA) and the mean diffusivity (MD). IVIM enabled the measurement of the contribution of microvascular perfusion (perfusion fraction, PF) to the diffusion coefficient $(D)$. Local perfusion was measured with ASL MRI using four different inversion times, allowing both quantification of perfusion and determination of arterial transit times (ATT). 2D phase contrast (2DPC) MRI was used to measure total blood flow through the renal artery.

From the $R_{2}^{*}$ map (BOLD) and the $T_{2}$ map, $R_{2}^{\prime}$ could be calculated:

$R_{2}^{\prime}=R_{2}^{*}-\frac{1}{T_{2}}$

where $R_{2}^{\prime}, R_{2}^{*}$ and $T_{2}$ are the median values of the parenchymal ROIs. Compared to $R_{2}^{*}, R_{2}^{\prime}$ is considered to reflect oxygenation more directly since it is not influenced by changes in $T_{2}$.

\section{Histology}

After fixation in formalin, coronal sections of the explanted kidney were obtained from the upper pole (ventral, dorsal and lateral side), lower pole (ventral, dorsal and lateral side) and hilar region (ventral and dorsal side). In addition, we added a nephrectomy sample from a tumor nephrectomy where we show the healthy tissue as comparison to the explanted graft. Sections were paraffin embedded. Next, slides were cut at $3 \mu \mathrm{m}$ and stained with hematoxylin and eosin (H\&E), periodic acid-Schiff (PAS) and methenamine silver (Jones' stain) using standard protocols. Finally, slides were analyzed by two experienced renal pathologists (RG, TQN).

\section{Statistical analysis}

MRI measures of the entire parenchyma were not normally distributed due to substantial differences between cortex and medulla in healthy kidneys. Therefore, median values and interquartile ranges (IQR) were calculated for the entire parenchyma ROI analysis. To assess changes in texture, local standard deviation (SD) maps were calculated where each voxel contains the $\mathrm{SD}$ of the $3 \times 3$ surrounding voxels. The median values within the ROI on these local SD maps were reported as well. For healthy subjects, the groupwise mean and SD were reported for all measures. Differences between the patient's values and normative values were expressed as percentage differences with respect to the normative values and in SDs of the normative values. Since only one patient was analyzed, no comparative statistical tests were performed.

To compare the voxel distribution within the parameter maps between healthy and the diseased kidney, histograms were used. For each examination in each subject, a histogram was calculated. In the controls, the median histogram was calculated. Parameter variations within controls were demonstrated by the IQR, the 10-90 and 2.5-97.5 interpercentile ranges, and are shown as shaded areas on the histograms. Results of histology were described qualitatively. The comparison between histopathology and MRI findings was performed in a qualitative way by an experienced renal pathologist (TQ) and an author with 5 years of experience in renal MRI $(\mathrm{AB})$.

\section{Results}

Data of 19 healthy subjects with a median age of 49 (IQR 45-57) were used as normative values. The patient tolerated the MRI examination well and all data were of sufficient quality to be included in the current analysis.

\section{MRI_comparison to normative values}

In Figs. 1 and 2, the MRI parameter maps belonging to the patient are shown alongside corresponding native kidney maps of a single control. For almost all MR measurements, noticeable differences could be appreciated between the patient and the control.

In healthy subjects, the cortex and medulla could be easily discriminated thanks to the higher $T_{1}$ in the medulla. Within the cortex and medulla, the $T_{1}$ was rather homogeneous. In the patient, however, there was no difference between cortex and medulla and the $T_{1}$ values within the parenchyma were heterogeneous (Fig. 1). However, due to the loss of corticomedullary differentiation, this was not reflected in the IQR (167 ms vs 213 (37) ms, Table 2) nor in the local SD (91 vs 96 (16) ms). Overall, $T_{1}$ was increased in the diseased kidney due to microstructural changes in the tissue, most likely fibrosis and influx of inflammatory cells (Table 2). 

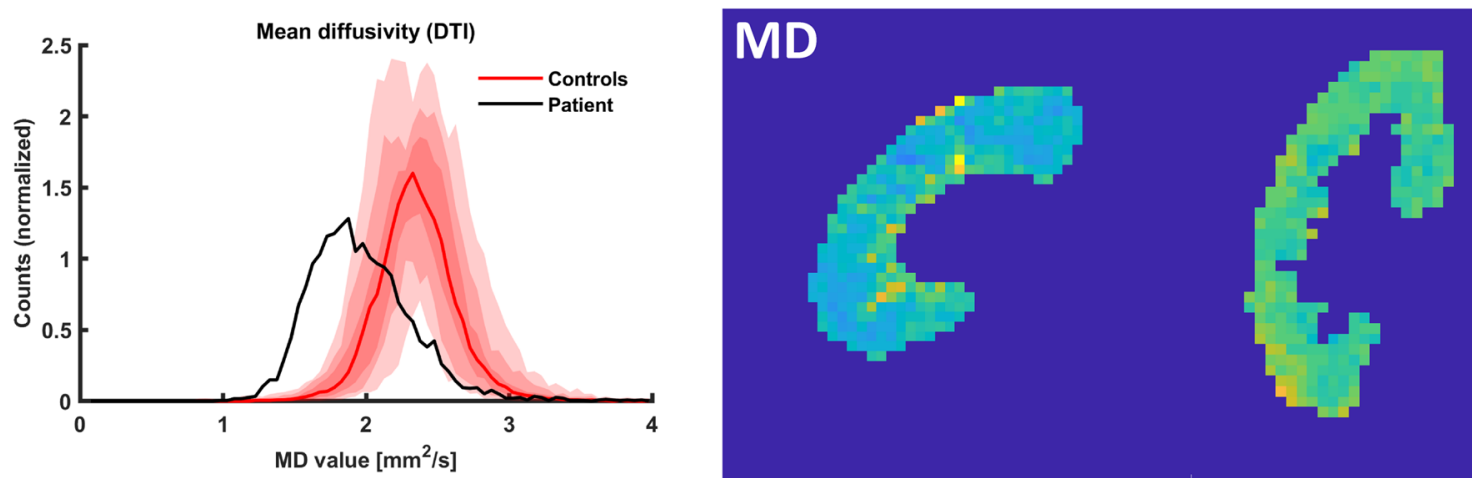

$2 \mathrm{~mm}^{2} / \mathrm{s}$
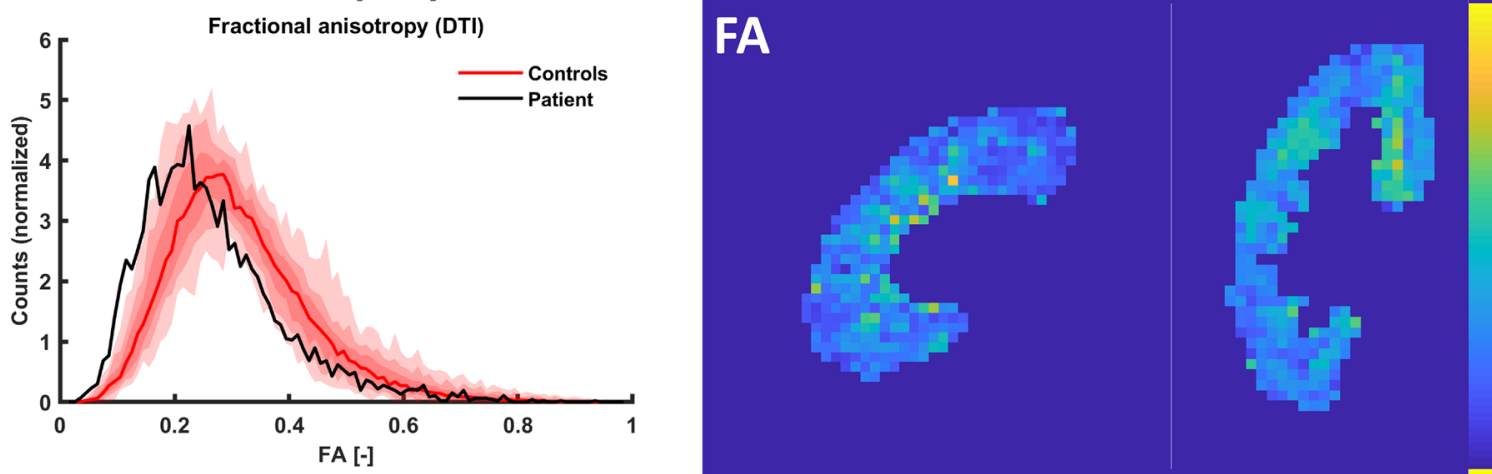

$0 \mathrm{~mm}^{2} / \mathrm{s}$
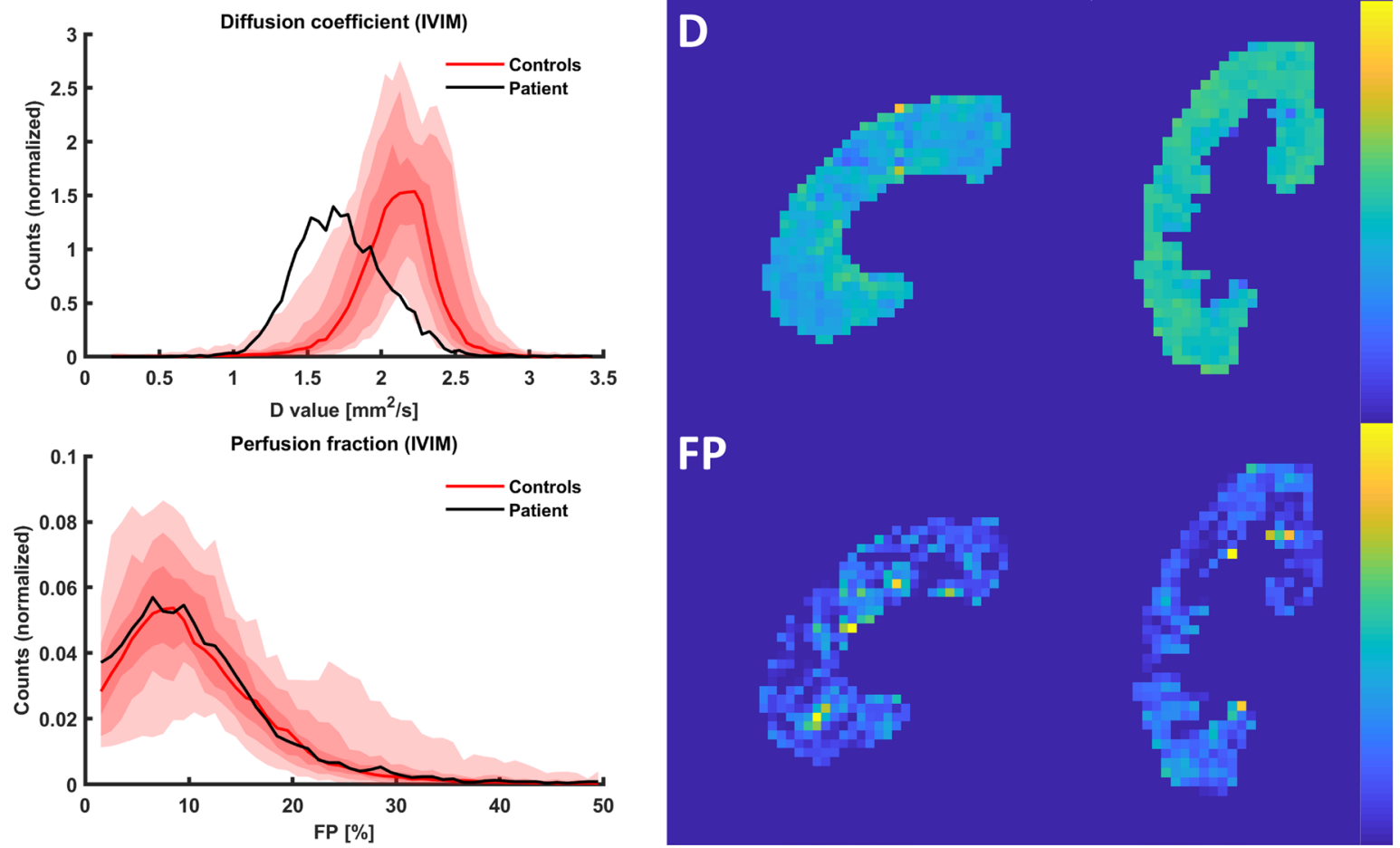

Fig. 2 Histograms (left) and parameter maps for the patient (middle column) and a control (right) for all diffusion measures: MD and FA from the DTI analysis and D and PF from the IVIM analysis. The histograms show the voxel distribution in the patient and the median voxel distribution in controls, along with the spread between controls. $M D$ mean diffusivity, $F A$ fractional anisotropy, $D$ diffusion coefficient, $P F$ perfusion fraction 
The parenchymal $T_{2}$ of the diseased kidney was decreased as compared to controls (Table 2). In Fig. 1, marked heterogeneity was seen throughout the parenchyma reflected by large increase in both local SD (14.5 vs $7.8(1.3) \mathrm{ms})$ and IQR (25 vs 15 (3) ms) in the diseased kidney. Regionally, $T_{2}$ approached $150 \mathrm{~ms}$, which is the upper cut-off value of the fit. $T_{2}$ values in this range indicate high water content, for example, edema or fluid collections. Due to fixation, this could not be confirmed histologically.

The BOLD or $R_{2}^{*}$ map confirms the finding of increased heterogeneity, which was also reflected in the broader histogram compared to the controls (Fig. 1), the increased IQR $\left(10.8\right.$ vs $\left.7.1(1.4) \mathrm{s}^{-1}\right)$ and the marked increase in local SD (3.9 vs $2.4(0.4) \mathrm{s}^{-1}$ ). No marked difference in global median $R_{2}^{*}$ value between the patient and healthy subjects was seen (Table 2).

Regarding perfusion, in healthy subjects, areas with high perfusion were located in the cortex, but in the patient, these areas were located more centrally, corresponding to the locations of large vessels (Fig. 3). Around patent large vessels, perfusion was very high, while other areas seemed devoid of any blood supply. In healthy subjects, the latter was only seen in the inner medulla. The arterial transit times also showed a heterogeneous pattern in the diseased kidney (IQR 0.99 vs 0.43 (0.15)), compared to homogeneous and relatively short arrival times in the healthy cortex. Overall, 2DPC showed a marked decrease in blood flow through the renal artery in the diseased kidney (140 vs 422 (159) mL/ $\min )$.

Results of the diffusion analysis are shown in Fig. 2. Both $\mathrm{MD}$ and $\mathrm{D}$ showed higher restriction of diffusion compared to healthy subjects (Table 2), consistent with fibrosis and inflammation. A slight increase in heterogeneity was seen in the patient in all diffusion measures. FA was slightly decreased indicating less anisotropy, while no clear difference was seen between the patient and the controls for the PF. However, it should be noted that the quality of PF map was lower than the other parameter maps.

\section{Histology-comparison to MRI}

Macroscopically, the explanted kidney was small $(10 \times 4.5 \times 4.5 \mathrm{~cm})$ and multiple hematomas were seen (Fig. 4a). Hematomas are expected to be visible at $R_{2}^{*}$ (BOLD) maps as areas with high $R_{2}^{*}$. The areas with $R_{2}^{*}$ close to $50 \mathrm{~s}^{-1}$ (the cut-off value) seen on the $R_{2}^{*}$ map correlated with the locations of hematomas (Fig. 4a, b).

Microscopic examination revealed extensive microstructural damage as compared to healthy histology (Supplemental Fig. 1). Most proximal vasculature, including the interlobar and arcuate arteries in the medulla, was still patent (Fig. 5a), while the distal vasculature including the interlobular arteries in the cortex were severely obliterated
Table 2 MRI measures for the patient compared to controls

\begin{tabular}{|c|c|c|c|c|}
\hline & Patient & Controls & Difference $(\%)$ & Difference (SD) \\
\hline \multicolumn{5}{|l|}{$T_{1} \operatorname{map}(\mathrm{ms})$} \\
\hline Median & 1732 & $1556(59)$ & +11 & +3.0 \\
\hline IQR & 167 & $213(37)$ & -21 & -1.2 \\
\hline Local SD & 91 & $96(16)$ & -5 & -0.3 \\
\hline \multicolumn{5}{|l|}{$T_{2}$ map $(\mathrm{ms})$} \\
\hline Median & 97 & $113(8)$ & -14 & -1.8 \\
\hline IQR & 25 & $15(3)$ & +67 & +3.9 \\
\hline Local SD & 14.5 & $7.8(1.3)$ & +87 & +5.1 \\
\hline \multicolumn{5}{|c|}{$R_{2}^{*} \mathrm{map} / \mathrm{BOLD}\left(\mathrm{s}^{-1}\right)$} \\
\hline Median & 21.7 & $19.0(2.1)$ & +15 & +1.3 \\
\hline IQR & 10.8 & $7.1(1.4)$ & +52 & +2.5 \\
\hline Local SD & 3.9 & $2.4(0.4)$ & +61 & +4.0 \\
\hline \multicolumn{5}{|c|}{$R_{2}^{\prime}\left(\mathrm{BOLD}\right.$ and $\left.T_{2} \mathrm{map}\right)\left(\mathrm{s}^{-1}\right)$} \\
\hline Median & 11.4 & $10.1(1.9)$ & +13 & +0.7 \\
\hline \multicolumn{5}{|c|}{ ASL-perfusion (ml/100 mL/min) } \\
\hline Median & 170 & $339(59)$ & -50 & -2.9 \\
\hline IQR & 359 & $140(35)$ & +155 & +6.3 \\
\hline Local SD & 172 & $79(45)$ & +118 & +2.1 \\
\hline \multicolumn{5}{|c|}{ ASL-ATT (s) } \\
\hline Median & 0.93 & $0.48(0.15)$ & +95 & +3.1 \\
\hline IQR & 0.99 & $0.43(0.15)$ & +128 & +3.7 \\
\hline Local SD & 0.45 & $0.20(0.13)$ & +122 & +2.0 \\
\hline \multicolumn{5}{|c|}{ 2DPC-blood flow (ml/min) } \\
\hline NA & 140 & $422(159)$ & -67 & -1.8 \\
\hline \multicolumn{5}{|c|}{ DTI-MD $\left(\mathrm{mm}^{2} / \mathrm{s}\right)$} \\
\hline Median & 1.9 & $2.4(0.1)$ & -19 & -3.6 \\
\hline IQR & 0.46 & $0.31(0.06)$ & +48 & +2.5 \\
\hline Local SD & 0.26 & $0.19(0.04)$ & +34 & +1.8 \\
\hline \multicolumn{5}{|c|}{ DTI-FA (fraction) } \\
\hline Median & 0.23 & $0.29(0.03)$ & -20 & -1.9 \\
\hline IQR & 0.15 & $0.15(0.02)$ & +1 & +0.1 \\
\hline Local SD & 0.10 & $0.09(0.01)$ & +1 & +0.1 \\
\hline \multicolumn{5}{|c|}{$\operatorname{IVIM}-D\left(\mathrm{~mm}^{2} / \mathrm{s}\right)$} \\
\hline Median & 1.7 & $2.1(0.1)$ & -20 & -2.9 \\
\hline IQR & 0.42 & $0.30(0.07)$ & +41 & +1.8 \\
\hline Local SD & 0.23 & $0.17(0.03)$ & +31 & +1.5 \\
\hline \multicolumn{5}{|c|}{ IVIM-PF (\%) } \\
\hline Median & 7.9 & $8.8(3.3)$ & -9 & -0.3 \\
\hline IQR & 10.5 & $10.3(3.1)$ & +1 & 0 \\
\hline Local SD & 5.6 & $5.2(1.2)$ & +8 & +0.3 \\
\hline
\end{tabular}

Median values, interquartile ranges and the local SDs of wholeparenchyma ROIs are reported. For the controls, the groupwise mean (standard deviation) of the ROI medians, IQRs and local SDs are calculated. Percentage difference between patient and controls with respect to the controls was calculated, as well as the absolute difference in units of the SD of the distribution in healthy volunteers

$I Q R$ interquartile range, $S D$ standard deviation, $B O L D$ blood oxygenation level-dependent MRI, $A S L$ arterial spin labeling, $A T$ arterial transit time, $2 D P C 2 \mathrm{D}$ phase contrast, $D T I$ diffusion tensor imaging, $M D$ mean diffusivity, FA fractional anisotropy, IVIM intravoxel incoherent motion, $D$ diffusion coefficient, $P F$ perfusion fraction 

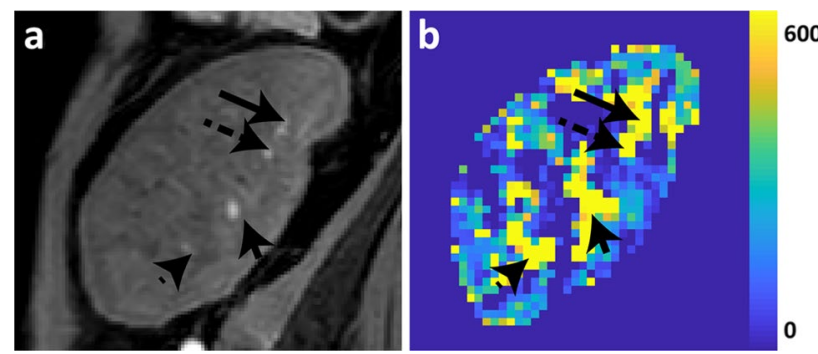

Fig. 3 Comparison of anatomical $T_{1}$-weighted Dixon (a) to perfusion map as obtained by ASL (b). In the Dixon, vessels have high signal thanks to inflow of fresh blood. On the perfusion map, vessels can be recognized as areas with very high flow $(\sim 600 \mathrm{~mL} / 100 \mathrm{~mL} / \mathrm{min})$. Because the spatial resolution of the Dixon is much higher compared to the perfusion map $(1 \times 1 \times 2 \mathrm{~mm}$ for the Dixon compared to $3 \times 3 \times 6 \mathrm{~mm}$ for the perfusion map), the vessels seem larger and more extensive on the perfusion map. Arrows show the locations of corresponding vessels on the Dixon and on the perfusion map

due to concentric intimal fibrosis with infiltration of plasma cells and lymphocytes (Fig. 5b). This was consistent with the perfusion maps obtained from ASL, which showed diminished perfusion of the parenchyma with very high perfusion around large vessels (Fig. 1).

Throughout the kidney but particularly at the dorsal side of the upper pole and lateral side, extensive areas with interstitial hemorrhages were seen (Fig. 5c). Most erythrocytes within these hemorrhages were intact and showed properties consistent with acute hemorrhage, although some scattered areas with hemosiderin-laden macrophages were found, indicative of older hemorrhage. The cortex was affected by severe glomerulosclerosis, tubular atrophy and interstitial fibrosis, and contained a dense inflammatory infiltrate
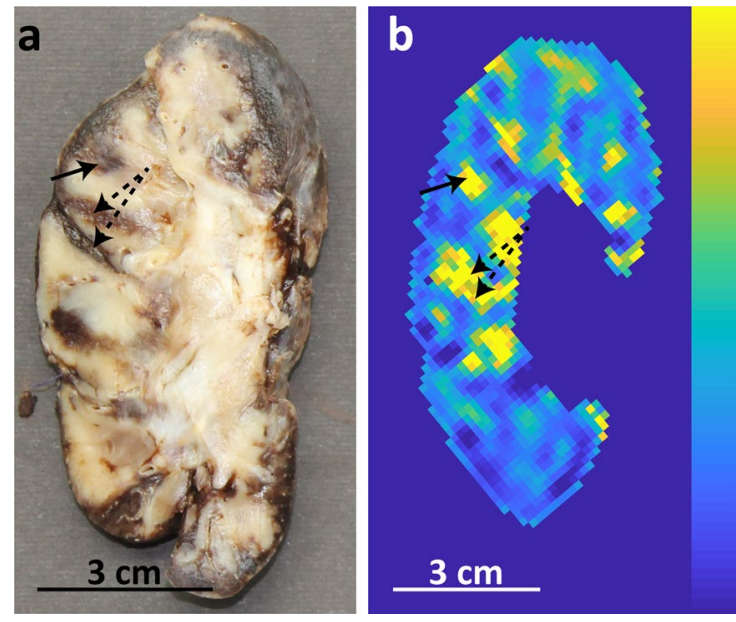

$50 \mathrm{~s}^{-1}$

Fig. 4 Macroscopic image of the explanted kidney (a) shown alongside the corresponding $R_{2}^{*}$ map (b). The $R_{2}^{*}$ map clearly shows the hemorrhages which are denoted by the arrows. The characteristic shape of the hemorrhage at the dashed arrows can also be recognized on the $R_{2}^{*}$ map
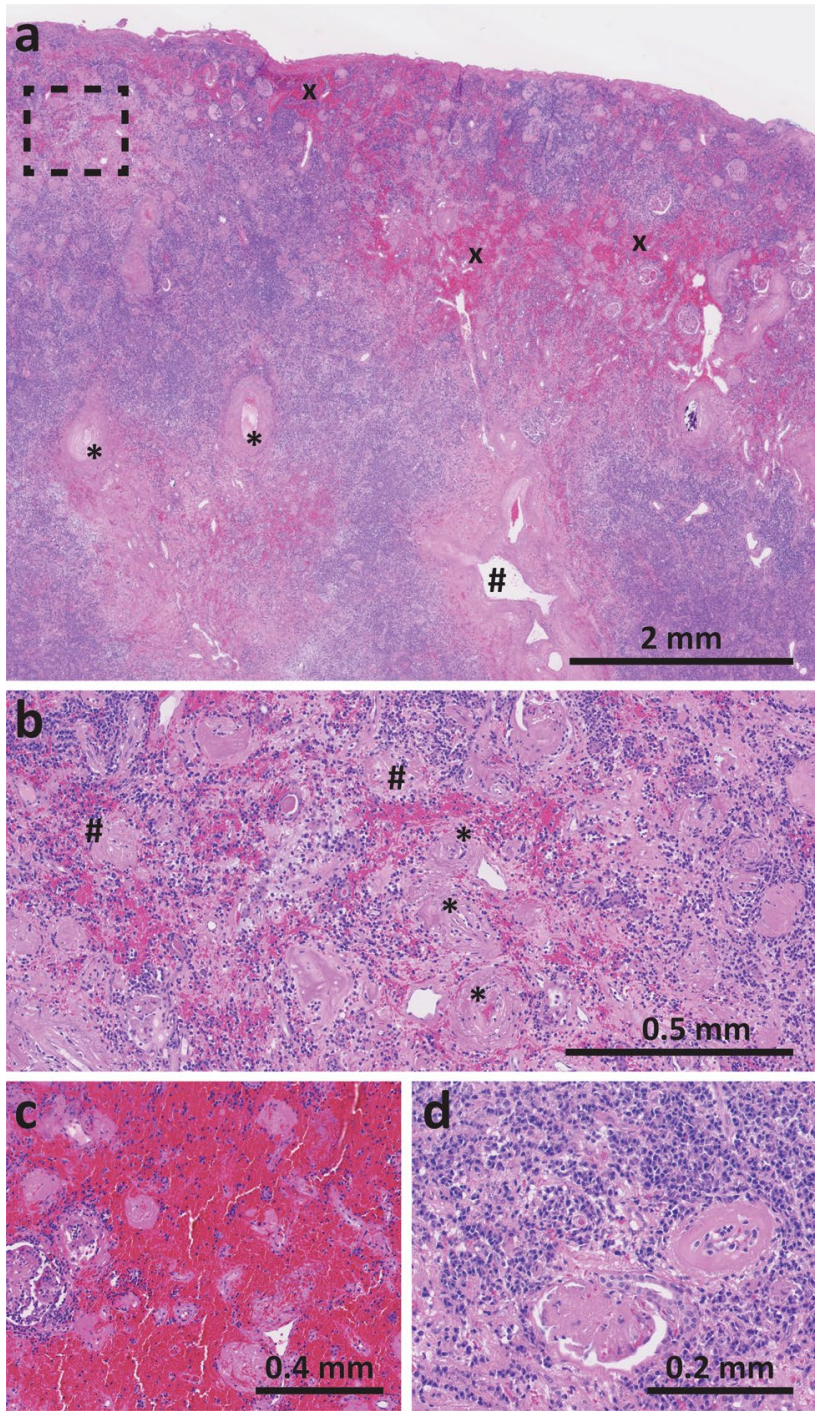

Fig. 5 Histology findings of the explanted kidney (all H\&E stain); a overview of cortex and medulla. The medulla contains patent interlobar arteries (\#) and arcuate arteries (*). Regions affected by hemorrhaging are denoted with an $\mathbf{X}$; $\mathbf{b}$ higher magnification of cortex annotated by the dashed rectangle in A showing completely obliterated interlobular arteries (*) and globally sclerosed glomeruli (\#); c cortical area with extensive interstitial hemorrhage; $\mathbf{d}$ cortical area with dense interstitial inflammatory infiltrate

consisting mainly of plasma cells (Fig. 5d). The medulla displayed a similar severity of inflammation. Although some non-sclerosed glomeruli were present focally, atrophy, inflammation and hemorrhage affected almost all proximal tubules. Those global changes in the renal parenchyma were in line with the MRI findings. These showed increased $T_{1}$ and restricted diffusion consistent with inflammatory infiltrate and fibrosis and a decreased $T_{2}$ which is consistent with fibrosis and hemorrhages. 


\section{Discussion}

In this study, we aimed to validate multiparametric MRI of a kidney transplant with end-stage disease to whole-kidney histology obtained after explantation. Multiparametric MRI findings were compared to normative values and to histology. Important clinical predictors of graft survival, IF/TA and capillary rarefaction could be measured with MRI. IF/TA resulted in restriction of diffusion as detected by a decrease in MD and D and in an increase in $T_{1}$. Capillary rarefaction could be identified by reduced parenchymal perfusion, which was detected as a decrease in total perfusion by ASL and 2DPC, as well as by a radical shift in perfusion distribution on ASL perfusion maps.

The combination of fibrosis and inflammation in the allograft parenchyma was reflected by increased $T_{1}$ and diffusion restriction $[12,13]$. On the other hand, $T_{2}$ was decreased, probably due to the severe fibrosis $[14,15]$ and the scattered hemorrhages since dominance of the widespread inflammation would have been expected to result in increase of $T_{2}$ [16].

Global changes in median $R_{2}^{*}$ and $R_{2}^{\prime}$ were minimal. However, heterogeneity of $R_{2}^{*}$ as measured with the local SD was markedly increased (+ 4.0 SD compared to controls). This probably was caused by the widespread fresh hemorrhages that caused local increases in $R_{2}^{*}$ values. While the hemorrhages also increased $R_{2}^{*}$ and $R_{2}^{\prime}$ on a global level, this was compensated by a decrease in $R_{2}^{*}$ (and $R_{2}^{\prime}$ ) due to capillary rarefaction which reduced blood volume and perfusion. Diminished oxygen demand in the virtually nonfunctioning kidney decreased deoxyhemoglobin synthesis as well, decreasing $R_{2}^{*}$ [17]. However, this was compensated by the oxygen demand of active inflammatory cells.

Perfusion of the diseased kidney was less than half of that measured in control subjects as demonstrated by both ASL and 2DPC. The large increase in median ATT indicated that arterial blood supply to the renal parenchyma was heavily delayed. Perfusion maps showed diminished parenchymal perfusion with maintained perfusion in the large vessels. This was consistent with histology showing patent interlobar and arcuate arteries, whereas the most distal interlobular arteries and arterioles were obliterated.

The obliteration of smaller vessels would have been expected to result in a decreased $\mathrm{PF}$ as measured with DWI [5]. In repeatability studies in healthy volunteers and patients, large variations in repeat measurements of $\mathrm{PF}$ have been observed $[5,9,18-20]$. The lack of difference in PF in might, therefore, be attributable to an unreliable measurement, which makes it currently less suitable for diagnostic purposes.

Changes in most MRI measurements in the diseased transplant kidney correlated well with pathological changes seen at macroscopic as well as microscopic analysis of the explanted allograft. Since the diseased allograft had been nonfunctioning for years prior to MRI and explantation, not all results of this study can be simply extrapolated to predict and interpret MRI findings in less damaged transplants. For example, the heterogeneity seen on virtually all MRI measurements was probably caused by scattered hemorrhages, which usually do not occur in earlier stages allograft nephropathy. The histological lesions that are routinely seen in less damaged transplants, such as fibrosis and inflammation, were present in an extreme form that is usually not seen in a functioning graft. However, the changes in $T_{1}$, diffusion coefficients and perfusion which we found are in line with the literature on chronic kidney disease both in native kidneys and transplants and probably do reflect the final stage of a continuous process. $T_{1}$, diffusion coefficients and perfusion measurements are promising non-invasive surrogate markers for severity of fibrosis and IF/TA, inflammation, and capillary rarefaction. However, for clinical diagnostic use of MRI, its lack of specificity remains a problem. The decrease in $T_{2}$ we found is interesting in this context since it is expected to change differently in response to inflammation then to fibrosis, but more research in renal disease is needed to confirm this.

A methodological limitation of this study was that the explanted kidney was sectioned at only three levels in the coronal plane (ventral, dorsal and lateral) of the upper and lower poles. These sections, therefore, did not exactly match the coronal plane of the MRI scans. This might have led to underestimation of the accuracy by which MRI scans can detect histological abnormalities. During explantation, superficial damage was inflicted to the kidney which might have caused superficial hemorrhaging and consequently might have influenced histology. In addition, the damage to the graft was to an extent usually not seen in healthy grafts. Although our findings are in line with previous studies on MRI in patients with chronic kidney disease, it is unknown how they will translate to less severely damaged grafts. However, if in such an extreme case no changes in MRI parameters could be observed, changes are likely also not observed in less severe cases. Furthermore, the MRI images were compared to images and normative values obtained in healthy volunteers, as opposed to patients with well-functioning transplants which would have been more appropriate, but this was beyond the scope of this study. Kidney transplantation is known to induce physiological and microstructural changes in the graft, which can be measured with MRI [5], so a comparison to well-functioning grafts might yield slightly different results. In future studies, we aim to make a comparison between ill-functioning and relatively healthy transplant kidneys. 
Currently, acquisition time of this multiparametric protocol was approximately $1 \mathrm{~h}$, which might be challenging to fit in clinical timeslots. Future studies will most likely determine which acquisitions provide clinically valuable information and which acquisitions do not contribute and can, therefore, be discarded. This will shorten acquisition time.

In conclusion, this patient gave us the unique opportunity to directly compare MR images obtained shortly before explantation with whole organ histology. Important histological predictors of long-term graft survival after kidney transplantation, like IF/TA and capillary rarefaction, could be identified with a combination of multiple functional MRI techniques, which underscores the high potential of the latter for non-invasive acquisition of valuable information for clinical decision-making.

Acknowledgements $\mathrm{AB}$ and TP were supported by an Alexandre Suerman stipend of the University Medical Center Utrecht, the Netherlands. $\mathrm{CB}$ and $\mathrm{AH}$ acknowledge funding from the Netherlands Organization for Scientific Research (14951).

Author contributions $\mathrm{AB}$ and $\mathrm{TP}$ contributed to study conception and design, acquisition, analysis and interpretation of data and drafting of the manuscript. PJB, HH, JAJ, MCV, TL and ADZ contributed to study conception and design and critical revision of the manuscript. AAH, $\mathrm{CB}, \mathrm{MF}, \mathrm{RG}, \mathrm{B}-\mathrm{JP}$, and TQN contributed to acquisition, analysis and interpretation of data and revision of the manuscript. All authors read and approved the final manuscript.

\section{Compliance with ethical standards}

Conflict of interest None declared.

Ethical approval This study was performed according to the declaration of Helsinki and ethical guidelines of our institution. The clinical and research activities being reported are consistent with the Principles of the Declaration of Istanbul as outlined in the 'Declaration of Istanbul on Organ Trafficking and Transplant Tourism'. The histology shown in this study was collected as part of routine clinical care. The protocol used in this study was approved by the institutional review board of our center. The patient signed informed consent for the acquisition of the MRI and use of the data. Control data were acquired as part of a separate study, which was approved be the institutional review board. All control subjects signed informed consent.

Open Access This article is licensed under a Creative Commons Attribution 4.0 International License, which permits use, sharing, adaptation, distribution and reproduction in any medium or format, as long as you give appropriate credit to the original author(s) and the source, provide a link to the Creative Commons licence, and indicate if changes were made. The images or other third party material in this article are included in the article's Creative Commons licence, unless indicated otherwise in a credit line to the material. If material is not included in the article's Creative Commons licence and your intended use is not permitted by statutory regulation or exceeds the permitted use, you will need to obtain permission directly from the copyright holder. To view a copy of this licence, visit http://creativecommons.org/licenses/by/4.0/.

\section{References}

1. Abecassis M, Bartlett ST, Collins AJ et al (2008) Kidney transplantation as primary therapy for end-stage renal disease: a National Kidney Foundation/Kidney Disease Outcomes Quality Initiative (NKF/KDOQITM) conference. Clin J Am Soc Nephrol 3(2):471-480. https://doi.org/10.2215/CJN.05021107

2. Josephson MA (2011) Monitoring and managing graft health in the kidney transplant recipient. Clin J Am Soc Nephrol 6(7):1774 1780. https://doi.org/10.2215/CJN.01230211

3. Brachemi S, Bollee G (2014) Renal biopsy practice: what is the gold standard? World J Nephrol 3(4):287-294. https://doi. org/10.5527/wjn.v3.i4.287

4. Selby NM, Blankestijn PJ, Boor P et al (2018) Magnetic resonance imaging biomarkers for chronic kidney disease: a position paper from the European Cooperation in Science and Technology Action PARENCHIMA. Nephrol Dial Transplant 33(suppl_2):ii4-ii14. https://doi.org/10.1093/ndt/gfy152

5. Bane O, Hectors SJ, Gordic S et al (2020) Multiparametric magnetic resonance imaging shows promising results to assess renal transplant dysfunction with fibrosis. Kidney Int 97(2):414-420. https://doi.org/10.1016/j.kint.2019.09.030

6. Friedli I, Crowe LA, Berchtold L et al (2016) New magnetic resonance imaging index for renal fibrosis assessment: a comparison between diffusion-weighted imaging and T1 mapping with histological validation. Sci Rep 6:30088. https://doi. org/10.1038/srep30088

7. Wang W, Yu Y, Wen J et al (2019) Combination of functional magnetic resonance imaging and histopathologic analysis to evaluate interstitial fibrosis in kidney allografts. Clin J Am Soc Nephrol 14(9):1372-1380. https://doi.org/10.2215/cjn.00020 119

8. Cutajar M, Thomas DL, Hales PW et al (2014) Comparison of ASL and DCE MRI for the non-invasive measurement of renal blood flow: quantification and reproducibility. Eur Radiol 24(6):1300-1308. https://doi.org/10.1007/s00330-014-3130-0

9. De Boer A, Harteveld AA, Stemkens B et al (2020) Multi-parametric renal MRI - an intra-subject test-retest repeatability study. J Magn Reson Imaging. https://doi.org/10.1002/jmri.27167

10. Cosio FG, El Ters M, Cornell LD, Schinstock CA, Stegall MD (2016) Changing kidney allograft histology early posttransplant: prognostic implications of 1-year protocol biopsies. Am J Transpl 16(1):194-203. https://doi.org/10.1111/ajt.13423

11. Steegh FMEG, Gelens MACJ, Nieman FHM et al (2011) Early loss of peritubular capillaries after kidney transplantation. J Am Soc Nephrol 22(6):1024. https://doi.org/10.1681/ASN.20100 50531

12. Caroli A, Schneider M, Friedli I et al (2018) Diffusion-weighted magnetic resonance imaging to assess diffuse renal pathology: a systematic review and statement paper. Nephrol Dial Transplant 33(suppl_2):ii29-ii40. https://doi.org/10.1093/ndt/gfy163

13. Wolf M, de Boer A, Sharma K et al (2018) Magnetic resonance imaging T1- and T2-mapping to assess renal structure and function: a systematic review and statement paper. Nephrol Dial Transplant 33(suppl_2):ii41-ii50. https://doi.org/10.1093/ndt/gfy198

14. Di Cesare E (2001) MRI of the cardiomyopathies. Eur J Radiol 38(3):179-184. https://doi.org/10.1016/s0720-048x(01)00311-4

15. Matsumoto S, Mori H, Miyake $\mathrm{H}$ et al (1998) MRI signal characteristics of progressive massive fibrosis in silicosis. Clin Radiol 53(7):510-514. https://doi.org/10.1016/s0009-9260(98)80171-2

16. Kim PK, Hong YJ, Im DJ et al (2017) Myocardial T1 and T2 mapping: techniques and clinical applications. Korean J Radiol 18(1):113-131. https://doi.org/10.3348/kjr.2017.18.1.113

17. Niendorf T, Pohlmann A, Arakelyan K et al (2015) How bold is blood oxygenation level-dependent (BOLD) magnetic resonance 
imaging of the kidney? Opportunities, challenges and future directions. Acta Physiol (Oxf) 213(1):19-38. https://doi.org/10.1111/ apha. 12393

18. Cox EF, Buchanan CE, Bradley CR et al (2017) Multiparametric renal magnetic resonance imaging: validation, interventions, and alterations in chronic kidney disease. Front Physiol 8:696. https ://doi.org/10.3389/fphys.2017.00696

19. Bane O, Wagner M, Zhang JL et al (2016) Assessment of renal function using intravoxel incoherent motion diffusion-weighted imaging and dynamic contrast-enhanced MRI. J Magn Reson Imaging 44(2):317-326. https://doi.org/10.1002/jmri.25171
20. Pan J, Zhang H, Man F et al (2018) Measurement and scan reproducibility of parameters of intravoxel incoherent motion in renal tumor and normal renal parenchyma: a preliminary research at 3.0 T MR. Abdom Radiol (NY) 43(7):1739-1748. https://doi. org/10.1007/s00261-017-1361-7

Publisher's Note Springer Nature remains neutral with regard to jurisdictional claims in published maps and institutional affiliations. 